\title{
Prevalenza, caratteristiche cliniche e biochimiche e risultati del trattamento dell'acromegalia con normali livelli di GH alla diagnosi
}

\author{
Salvatore Cannavò ${ }^{1}$
}

Pubblicato online: 4 dicembre 2018

(c) Springer Nature Switzerland AG 2018

Commento a:

Prevalence, clinical and biochemical spectrum and treatment outcome of acromegaly with normal basal GH at diagnosis.

A.L. Espinosa-de-los-Monteros, E. Sosa-Eroza, B. Gonzalez, V. Mendoza, M. Mercado.

J Clin Endocrinol Metab (2018) https://doi.org/10.1210/ jc.2018-01113

Il termine di micromegalia è utilizzato per indicare una forma di acromegalia caratterizzata da elevati livelli di IGF-1, presenza di un adenoma ipofisario GH-secernente e contesto clinico appropriato, a fronte di valori di GH basali apparentemente normali e adeguatamente sopprimibili $(<1 \mathrm{ng} / \mathrm{ml})$ durante carico orale di glucosio $(75 \mathrm{gr})$. Tale condizione è stata descritta per la prima volta da Dimaraki et al. nel 2002 [1] e riguarda certamente una minoranza dei pazienti affetti da acromegalia, sebbene Butz et al. [2] abbiano recentemente evidenziato come il riscontro di valori discrepanti di GH e IGF-1 sia empre più frequente in pazienti con sospetta acromegalia.

In questo studio, Espinosa-de-los-Monteros et al. hanno valutato la prevalenza, le caratteristiche cliniche e biochimiche, le complicanze e l'outcome dell'approccio chirurgico in un gruppo di pazienti con micromegalia in confronto a pazienti con acromegalia classica. A tal fine, hanno suddiviso 528 pazienti con acromegalia in 4 gruppi, in base ai valori di GH random misurati al momento della diagnosi: nel gruppo 1 erano inclusi 16 pazienti con valori di $\mathrm{GH}<2 \mathrm{ng} / \mathrm{ml}$, il gruppo 2 era composto da 202 pazienti con valori di GH

S. Cannavò

cannavos@unime.it

1 Dipartimento di Patologia Umana dell'Adulto e dell'Età Evolutiva “G. Barresi”, Università di Messina, Messina, Italia fra 2 e $9,9 \mathrm{ng} / \mathrm{ml}$, il gruppo 3 da 294 pazienti con livelli di $\mathrm{GH}$ fra 10 e $99 \mathrm{ng} / \mathrm{ml}$, mentre il gruppo 4 era costituito da 16 pazienti con livelli di $\mathrm{GH}>100 \mathrm{ng} / \mathrm{ml}$. I pazienti del primo gruppo rappresentavano quindi il 3\% della casistica complessiva. Essi erano significativamente più anziani rispetto a quelli degli altri gruppi e il sesso maschile era prevalente rispetto al sesso femminile, contrariamente a quanto osservato nel resto della casistica. La frequenza di segni, sintomi e comorbilità tipiche dell'acromegalia erano simili nei quattro gruppi, ma nel gruppo 1 vi era una più elevata prevalenza di microadenomi ipofisari $(75 \%)$ e i valori medi di IGF-1 e di $\mathrm{GH}$, sia basale che post-OGTT, erano significativamente più bassi. Infine, la frequenza di successo chirurgico nei pazienti del gruppo $1(53,8 \%)$ non differiva da quella del gruppo $2(54,1 \%)$ e $3(369 \%)$ ma era significativamente più elevata rispetto al gruppo $4(13,3 \%)$, che era composto da pazienti con macroadenoma invasivo e/o gigante.

In conclusione, secondo questo studio la micromegalia non rappresenterebbe una forma di acromegalia diagnosticata più precocemente $\mathrm{o}$ meno grave rispetto alle forme classiche, ma solo una rara condizione riscontrata più frequentemente in pazienti anziani e di sesso maschile.

\section{Bibliografia}

1. Dimaraki EV, Jaffe CA, DeMott-Friberg R et al (2002) Acromegaly with apparently normal GH secretion: implication for diagnosis and follow-up. J Clin Endocrinol Metab 87:3537-3542

2. Butz LB, Sullivan SE, Chandler WF, Barkan AL (2016) "Micromegaly": an update on the prevalence of acromegaly with apparently normal GH secretion in the modern era. Pituitary 19:547-551 